\title{
Non-coherent sequence detection scheme for satellite-based automatic identification system
}

\author{
Haosu Zhou and Jianxin Wang* \\ School of Electronic and Optical Engineering, Nanjing University of Science and Technology, Nanjing 210094, China
}

\begin{abstract}
The satellite-based automatic identification system (AIS) receiver has to encounter the frequency offset caused by the Doppler effect and the oscillator instability. This paper proposes a non-coherent sequence detection scheme for the satellite-based AIS signal transmitted over the white Gaussian noise channel. Based on the maximum likelihood estimation and a Viterbi decoder, the proposed scheme is capable of tolerating a frequency offset up to $5 \%$ of the symbol rate. The complexity of the proposed scheme is reduced by the state-complexity reduction, which is based on per-survivor processing. Simulation results prove that the proposed non-coherent sequence detection scheme has high robustness to frequency offset compared to the relative scheme when messages collision exists.
\end{abstract}

Keywords: non-coherent sequence detection scheme, satellitebased automatic identification system, frequency offset, messages collision, Viterbi decoder.

DOI: $10.21629 /$ JSEE.2017.03.04

\section{Introduction}

The automatic identification system (AIS) is a universal ship tracking system, and its purpose is to provide efficient data exchange among ships and coast stations, thereby improve the general safety of navigation [1]. The exchanged different types of data include static information (identification, name of ship), dynamic information (position, heading, speed), voyage related information, and safety related information [2]. A self organizing time division multiple access (SOTDMA) scheme is employed by the AIS to quickly prevent against conflicts without intervention from controlling stations. Each user broadcasts its message in a dedicated time slot, which ensures the reliable information exchange of AIS equipments within radio visibility about 40 nautical miles [3]. Whereas, the shipto-coast station communications are difficult for ships at very large distances from coastline (more than 40 nautical miles). Receiving messages from a constellation of low

Manuscript received June 28, 2016.

*Corresponding author. earth orbit (LEO) satellites is a solution to this problem, ensuring a global coverage.

However, several technology issues which are excluded in an original AIS standard arise in a satellite-based receiver, where Doppler effect and message collision are two representative issues [4]. (i) The Doppler shift is a function of the relative velocity between the ship and the satellite. The ship velocity is small compared to the satellite velocity, such that the Doppler shift can be calculated as $\Delta f=v_{r} / \lambda$, where $\lambda$ denotes the wavelength of the original AIS signal and $v_{r}$ represents the element of the satellite velocity directed toward the ship. For typical LEO altitudes $(600 \mathrm{~km})$ and field of view used in AIS applications, the maximum Doppler shift is around $4 \mathrm{kHz}$. Then, the Doppler shift is in the range of $[-4 \mathrm{kHz}, 4 \mathrm{kHz}]$ due to the symmetry of the coverage area. Thus, the maximum relative Doppler shift between two messages is about $8 \mathrm{kHz}$ [4]. (ii) A satellite-based AIS equipment would receive uncoordinated messages from ships belonging to different SOTDMA regions. This is because that the satellite antenna footprint is much larger than the coverage of a typical SOTDMA region, and thus includes many ships which could transmit messages in the same time-slot. This intra-system interference occurring among ships that are not in terrestrial radio-frequency visibility is the bottleneck in the detection capability of a satellite-based receiver.

The satellite-based AIS reception has been discussed in previous works. Coherent sequence detection scheme and non-coherent sequence detection (NSD) scheme are two strategies for the satellite-based AIS sequence detection. A coherent scheme based on the maximum likelihood estimation and a Viterbi decoder provides a good demodulation performance [5]. Prevost et al. proposed an innovative coherent scheme with impressive performance improvement by using the cyclic redundancy check (CRC) to correct error bits [6]. Performances of these coherent schemes rely on accurate parameters estimation and tracking. However, it is difficult to recover and track the car- 
rier phase when messages collision exists. Non-coherent detection, robust to phase and oscillator instability, is an attractive strategy. Besides, the performance of the NSD scheme approaches that of the coherent scheme. Based on [7], an advanced NSD scheme was proposed by utilizing three zonal demodulators to process different (but overlapping) frequency bandwidths [8]. In order to improve the performance of non-coherent detection, Zhou et al. proposed a huge complexity scheme by using the CRC trellis error correction [9]. Compared to coherent schemes, they exhibited a negligible performance degradation [8-10] as long as the uncompensated frequency offset did not exceed an order of $1 \%$ of the symbol rate, whereas, for higher values, the performance rapidly degraded [11].

In this paper, we propose a new NSD scheme characterized by high robustness to frequency offset. Performance of the proposed scheme is practically limited by the matched-filter front end in the presence of a frequency offset. With the growth of frequency offset, the proposed scheme will have a moderate degradation with respect to the performance exhibited by the NSD scheme in [8]. From a practical point of view, frequency offsets up to $5 \%$ of the symbol rate can be tolerated. Besides, the complexity of the proposed detection is reduced with the statecomplexity reduction technology based on per-survivor processing [12].

This paper is organized as follows. Section 2 presents AIS concepts about the packet format, the Gaussian minimum shift-keying (GMSK) modulation, and the received AIS signal model. In Section 3, the NSD with high robustness to frequency offset is proposed. The complexity analysis of the proposed Viterbi detection is also presented. Section 4 gives some convincing simulation results of the proposed scheme compared to the detection scheme in [8]. Finally, Section 5 outlines some concluding remarks.

\section{AIS concepts}

\subsection{Packet format}

The data of the AIS signal is transferred using a transmission packet as shown in Table 1 [13].

Table 1 Packet format bit

\begin{tabular}{cccccc}
\hline Training sequence & Start flag & Data & FCS & End flag & Buffer \\
\hline 24 & 8 & 168 & 16 & 8 & 24 \\
\hline
\end{tabular}

The training sequence consists of alternating 0's and 1's $(010101 \cdots)$. Twenty-four bits of preamble are transmitted prior to sending the flag. The start flag, 8 bits long (01111110), consists of a standard high level data link control (HDLC) flag. It is used to detect the start of a transmission packet. The data portion is 168 bits long in the default transmission packet. The content of data is undefined at the data link service. The frame check sequence (FCS) uses the CRC 16-bit polynomial to calculate the checksum as defined in IOS/IEC 3309: 1993. The CRC bits should be pre-set to one at the beginning of a CRC calculation. Only the data portion should be included in the CRC calculation. The end flag is identical to the start flag.

The buffer is normally 24 bits and should be used as follows: bit stuffing-4 bits, distance delay-12 bits, repeater delay- 2 bits, and synchronization jitter- 6 bits. These stuffed bits, which can be used for limiting the number of consecutive bits of the same value in order to create additional signal transitions, are non-informative bits that can be inserted into the data of interest. These additional transitions are inserted to re-synchronize the receiver clock or to avoid specific code words. For example, the HDLC data transmission protocol uses bit stuffing to avoid the end frame flag byte in the data.

\subsection{GMSK modulation}

The output of the bit stuffing procedure is encoded with the non-return-to-zero inverted (NRZI) coding. The resulting sequence is modulated with GMSK modulation. The constant-modulus signal $s(t)$ is defined as

$$
s(t)=\mathrm{e}^{\mathrm{j} \varphi(t)}
$$

where the phase $\varphi(t)$ contains the information symbols. $\varphi(t)$ is defined as

$$
\varphi(t)=2 \pi h \sum_{n=-\infty}^{\infty} a_{n} \int_{-\infty}^{t} g\left(\tau-n T_{b}\right) \mathrm{d} \tau
$$

where $T_{b}$ is the symbol period, $\left\{a_{n}\right\}$ is the bit sequence belonging to the $M$-ary alphabet $(M=2), h=0.5$ is the modulation index, and for GMSK signals, the pulse $g(t)$ is defined as

$$
\begin{gathered}
g(t)=\frac{1}{2 T_{b}}\left\{Q\left[\frac{2 \pi B}{\sqrt{\ln 2}}\left(t-\frac{T_{b}}{2}\right)\right]-\right. \\
\left.Q\left[\frac{2 \pi B}{\sqrt{\ln 2}}\left(t+\frac{T_{b}}{2}\right)\right]\right\}
\end{gathered}
$$

where $B$ is the $3 \mathrm{~dB}$ cut-off bandwidth of the Gaussian low-pass filter, and

$$
Q(x)=\frac{1}{\sqrt{2 \pi}} \int_{x}^{\infty} \mathrm{e}^{-\frac{\tau^{2}}{2}} \mathrm{~d} \tau .
$$

The pulse $g(t)$ is generally truncated in the interval $\left[-L T_{b} / 2, L T_{b} / 2\right]$, and $L=3$.

Based on the Laurent representation, the complex envelope of AIS signals could be exactly expressed as

$$
s(t)=\sum_{k=0}^{K-1} \sum_{n} \alpha_{k, n} h_{k}\left(t-n T_{b}\right)
$$


where $K=(M-1) 2^{(L-1) \log _{2} M}$ is the number of linearly modulated pulses $\left\{h_{k}(t)\right\}$, and $\left\{\alpha_{k, n}\right\}$ are the so-called pseudo-symbols [14]. Most of the signal power is concentrated in the first $M-1=1$ component. We obtain the approximation as

$$
\begin{gathered}
s(t)=\sum_{n} \alpha_{0, n} h_{0}\left(t-n T_{b}\right) \\
\alpha_{0, n}=\mathrm{e}^{\mathrm{j} \frac{\pi}{2} \sum_{i=1}^{n} a_{i}} \\
h_{0}(t)=\prod_{i=0}^{L-1} u\left(t+i T_{b}\right)
\end{gathered}
$$

where

$$
\begin{gathered}
u(t)=\left\{\begin{array}{l}
\sin (\pi q(t)), \quad 0 \leqslant t \leqslant L T_{b} \\
u\left(2 L T_{b}-t\right), \quad L T_{b}<t \leqslant 2 L T_{b} \\
0, \quad \text { otherwise }
\end{array}\right. \\
q(t)=\int_{-\infty}^{t} g(\tau) \mathrm{d} \tau .
\end{gathered}
$$

\subsection{Received AIS signal model}

The signal received by the satellite could be expressed as

$$
\begin{gathered}
r(t)=A s(t-\tau) \mathrm{e}^{\mathrm{j}\left(2 \pi f_{d} t+\theta\right)}+ \\
\sum_{i=1}^{N_{I}} A_{i} s_{i}\left(t-\tau_{i}\right) \mathrm{e}^{\mathrm{j}\left(2 \pi \Delta f_{i} t+\theta_{i}\right)}+n(t)
\end{gathered}
$$

where $N_{I}$ is the number of interference signals, $s(t)$ is the signal of interest, $s_{i}(t)$ is the interference signals, $A$ and $A_{i}$ represent channel gains, $\tau$ and $\tau_{i}$ are time delays, $f_{d}$ and $\Delta f_{i}$ denote frequency offsets, $\theta$ and $\theta_{i}$ are phase shifts with the uniform distribution in the interval $[0,2 \pi)$ and $n(t)$ is a complex-valued white Gaussian noise process with independent components, each with two-sided power spectral density $N_{0}$. Frequency offset is made up of two parts. One part is the Doppler effect, and the other is the difference between the local oscillators.

\section{Proposed NSD scheme for satellite-based AIS}

In this section, we propose a new NSD scheme for the satellite-based AIS, which is robust to the high frequency offset. The structure of the proposed NSD scheme is depicted in Fig. 1. The proposed NSD scheme does not need to recover channel gain, frequency offset, and carrier phase of the interested signal.

Assume that the parameter time delay is known, then the received AIS signal without interference could be expressed as

$$
r(t)=A s(t) \mathrm{e}^{\mathrm{j}\left(2 \pi f_{d} t+\theta\right)}+n(t) .
$$

The received signal passes through a filter matched to $h_{0}(t)$ pulse. The output, sampled at time $n T_{b}$, of the matched filter can be expressed as

$$
x_{n}=\left.r(t) \otimes h_{0}(-t)\right|_{t=n T_{b}}
$$

where $\otimes$ denotes the convolution. The sample $x_{n}$ is sent to a whitening filter (WF), whose output could be written as

$$
z_{n}=\sum_{l=0}^{L_{w}-1} w_{l} x_{n-l}
$$

where $L_{w}$ is the length of the discrete-time whitening filter, and $\left\{w_{l}\right\}_{l=0}^{L_{w}-1}$ is the coefficients.

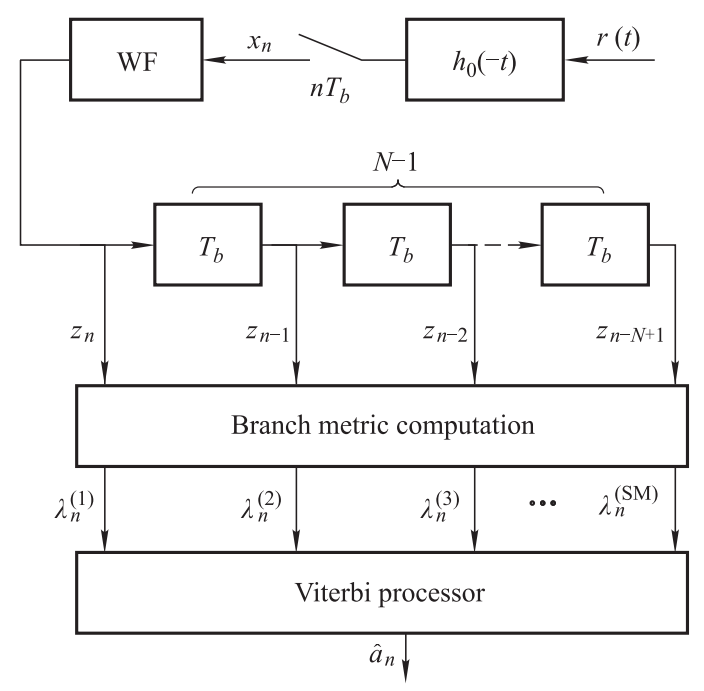

Fig. 1 Proposed NSD structure for satellite-based AIS

Colavolpe et al. proposed a frequency offset insensitive detection for linear modulation signals, which is based on the likelihood function for joint data detection and frequency offset estimation [11]. In this paper, we extend the likelihood function to the detection of AIS signals (non-linear modulation) without frequency offset estimation. The likelihood function is expressed as

$$
\begin{gathered}
\Gamma_{n}\left(\widetilde{\boldsymbol{a}}, \widetilde{f}_{d}\right)=N_{0} \ln I_{0}\left(\frac{1}{N_{0}}\left|\sum_{k=0}^{n-1} z_{k} \widetilde{y}_{k}^{*} \mathrm{e}^{-\mathrm{j} 2 \pi k \widetilde{f}_{d} T_{b}}\right|\right)- \\
\frac{1}{2} \sum_{k=0}^{n-1}\left|\widetilde{y}_{k}\right|^{2}
\end{gathered}
$$

where the expression of $\widetilde{y}_{k}$ could be found in [7] (note that $\left|\widetilde{y}_{k}\right|^{2}$ is not a constant), and $I_{0}(x)$ is the zeroth order modified Bessel function of the first kind. Since $\ln I_{0}(x) \approx x$, $\Gamma_{n}\left(\widetilde{\boldsymbol{a}}, \widetilde{f}_{d}\right)$ can be well approximated as

$$
\Gamma_{n}\left(\widetilde{\boldsymbol{a}}, \widetilde{f}_{d}\right)=\Gamma_{n}^{1}\left(\widetilde{\boldsymbol{a}}, \widetilde{f}_{d}\right)-\frac{1}{2} \sum_{k=0}^{n-1}\left|\widetilde{y}_{k}\right|^{2}
$$

where 


$$
\begin{gathered}
\Gamma_{n}^{1}\left(\widetilde{\boldsymbol{a}}, \widetilde{f}_{d}\right)=\left|\sum_{k=0}^{n-1} z_{k} \widetilde{y}_{k}^{*} \mathrm{e}^{-\mathrm{j} 2 \pi k \widetilde{f}_{d} T_{b}}\right|=\sqrt{\left|\sum_{k=0}^{n-1} z_{k} \widetilde{y}_{k}^{*} \mathrm{e}^{-\mathrm{j} 2 \pi k \widetilde{f}_{d} T_{b}}\right|^{2}}=\sqrt{\sum_{k=0}^{n-1} \sum_{m=0}^{n-1} z_{k} \widetilde{y}_{k}^{*} z_{m}^{*} \widetilde{y}_{m} \mathrm{e}^{-\mathrm{j} 2 \pi(k-m) \widetilde{f}_{d} T_{b}}}= \\
\sqrt{\frac{2 \operatorname{Re}\left\{\sum_{k=1}^{n-1} z_{k} \widetilde{y}_{k}^{*} \sum_{m=0}^{k-1} z_{m}^{*} \widetilde{y}_{m} \mathrm{e}^{\left.-\mathrm{j} 2 \pi(k-m) \widetilde{f}_{d} T_{b}\right\}}+\sum_{k=0}^{n-1}\left|z_{k} \widetilde{y}_{k}^{*}\right|^{2}\right.}{2 \sum_{i=1}^{n-1} \operatorname{Re}\left\{\sum_{k=i}^{n-1} z_{k} \widetilde{y}_{k}^{*} z_{k-i}^{*} \widetilde{y}_{k-i} \mathrm{e}^{-\mathrm{j} 2 \pi i \widetilde{f}_{d} T_{b}}\right\}+\sum_{k=0}^{n-1}\left|z_{k} \widetilde{y}_{k}^{*}\right|^{2}}}
\end{gathered}
$$

Note that $\operatorname{Re}\{x\}=|x| \cos (\arg (x))$. Then $\Gamma_{n}\left(\widetilde{\boldsymbol{a}}, \widetilde{f}_{d}\right)$ could be rewritten as

$$
\begin{aligned}
& \qquad \Gamma_{n}\left(\widetilde{\boldsymbol{a}}, \widetilde{f}_{d}\right)=\sqrt{2 \sum_{i=1}^{n-1}\left|\sum_{k=i}^{n-1} z_{k} \widetilde{y}_{k}^{*} z_{k-i}^{*} \widetilde{y}_{k-i}\right| \cos \left(\alpha_{i}(\boldsymbol{z}, \widetilde{\boldsymbol{y}})-2 \pi i \widetilde{f}_{d} T_{b}\right)+\sum_{k=0}^{n-1}\left|z_{k} \widetilde{y}_{k}^{*}\right|^{2}-\frac{1}{2} \sum_{k=0}^{n-1}\left|\widetilde{y}_{k}\right|^{2}} \\
& \qquad \alpha_{i}(\boldsymbol{z}, \widetilde{\boldsymbol{y}})=\arg \left\{\sum_{k=i}^{n-1} z_{k} \widetilde{y}_{k}^{*} z_{k-i}^{*} \widetilde{y}_{k-i}\right\} \quad \sum_{k=0}^{N-1}\left|z_{n-k} \widetilde{y}_{n-k}^{*}\right|^{2} \\
& \text { where } \boldsymbol{z}=\left[z_{0}, \ldots, z_{n-1}\right] \text {, and } \widetilde{\boldsymbol{y}}=\left[\widetilde{y}_{0}, \ldots, \widetilde{y}_{n-1}\right] . \text { In } \\
& \text { the absence of noise, the generic term } \alpha_{i}(\boldsymbol{z}, \widetilde{\boldsymbol{y}}) \text { is equal to } \\
& \begin{array}{l}
2 \pi i f_{d} T_{b} \text { for } \widetilde{\boldsymbol{y}}=\boldsymbol{y} \text {. Thus, all cosine factors in }(18) \text { are uni- } \\
\text { tary for } \widetilde{f}_{d}=f_{d} \text { and } \widetilde{\boldsymbol{y}}=\boldsymbol{y} \text {. Then, for data detection, we }
\end{array}
\end{aligned}
$$
define a partial sequence metric at the $n$th signaling interval

$$
\begin{gathered}
\Lambda_{n}(\widetilde{\boldsymbol{a}})=\Gamma_{n}\left(\widetilde{\boldsymbol{a}}, f_{d}\right) \approx \Lambda_{n}^{1}(\widetilde{\boldsymbol{a}})-\frac{1}{2} \sum_{k=0}^{n-1}\left|\widetilde{y}_{k}\right|^{2} \\
\Lambda_{n}^{1}(\widetilde{\boldsymbol{a}})=\sqrt{2 \sum_{i=1}^{n-1}\left|\sum_{k=i}^{n-1} z_{k} \widetilde{y}_{k}^{*} z_{k-i}^{*} \widetilde{y}_{k-i}\right|+\sum_{k=0}^{n-1}\left|z_{k} \widetilde{y}_{k}^{*}\right|^{2}}
\end{gathered}
$$

and an incremental or branch metric

$$
\lambda_{n}(\widetilde{\boldsymbol{a}})=\Lambda_{n+1}(\widetilde{\boldsymbol{a}})-\Lambda_{n}(\widetilde{\boldsymbol{a}})=\Lambda_{n+1}^{1}(\widetilde{\boldsymbol{a}})-\Lambda_{n}^{1}(\widetilde{\boldsymbol{a}})-\frac{1}{2}\left|\widetilde{y}_{n}\right|^{2} .
$$

A truncation is introduced to limit the memory of the incremental metric (22). We may only consider $N<<n$ most recent observations $z_{k}$ and code symbols $\widetilde{y}_{k}$, with $N$ being the implicit phase memory. For $n \geqslant N$, the truncatedmemory incremental, or branch metric approximates

$$
\lambda_{n}(\widetilde{\boldsymbol{a}})=\sqrt{\lambda_{n}^{1}}-\sqrt{\lambda_{n}^{2}}-\frac{1}{2}\left|\widetilde{y}_{n}\right|^{2}
$$

where

$$
\lambda_{n}^{1}=2 \sum_{i=1}^{N-1}\left|\sum_{m=0}^{N-i-1} z_{n-m} \widetilde{y}_{n-m}^{*} z_{n-m-i}^{*} \widetilde{y}_{n-m-i}\right|+
$$

According to the branch metric (23), the sequence $\left\{a_{n}\right\}$ will be detected by the Viterbi processor, which relies on a trellis state table and a trellis state transition table.

The number of trellis states and state transitions affects the complexity of the proposed scheme. The trellis state number of the proposed scheme is $S=2^{N_{s}-1}$, where $N_{s}=N+2$. The number of trellis states could be reduced by using state-complexity reduction [12]. In this paper, the reduced trellis state number is $S=2$. In order to compute the branch metrics (23) in a reduced trellis, the necessary symbols not included in the state definition could be found in the survivor history [15]. The training sequence and the start flag, known to the receiver, could be regarded as the initial survivors. The trellis must be constructed for the 216 symbols following the start flag of the AIS message. The trellis has two transitions starting from each state yielding $2 S$ transitions per symbol. For each transition, there are $3 N(N+1)+1$ multiplications and $N(N-1)+2$ additions for calculating $\lambda_{n}(\widetilde{\boldsymbol{a}})$. In other words, there are $4 N^{2}+2 N+3$ operations per transition. Thus, the total number of operations is $216 \times 2 S \times\left(4 N^{2}+2 N+3\right)$.

\section{Numerical results}

In this section, performance of the proposed NSD scheme is evaluated by simulations, in terms of bit error rate (BER) 
and packet error rate (PER). Each message consists of 168 bits concatenated with CRC, as described in Section 2.1. A stuffing bit 0 is inserted after each sequence of five consecutive bits 1 . The resulting sequence is encoded with NRZI, and then modulated with GMSK modulation. We consider AIS with parameters $B T_{b}=0.4$, and the symbol rate is $R_{b}=9600 \mathrm{bps}$. The proposed scheme is compared with the NSD scheme in [8], which is considered as the reference scheme for its better sequence detection performance. These schemes demodulate the received AIS signals without frequency offset estimation. The case of two messages collision is also under consideration in the simulations. The signal with lager channel gain is regarded as the interested signal, and the other is treated as the interference.

We consider the case of no interference in Fig. 2, which reports the performances of proposed receiver with different implicit phase memories in terms of BER and PER versus $E_{b} / N_{0}$, with $E_{b}$ being the received signal energy per information bit and $N_{0}$ being the noise power spectral density. The frequency offset of the received signal is $f_{d}=0 \% R_{b}$.

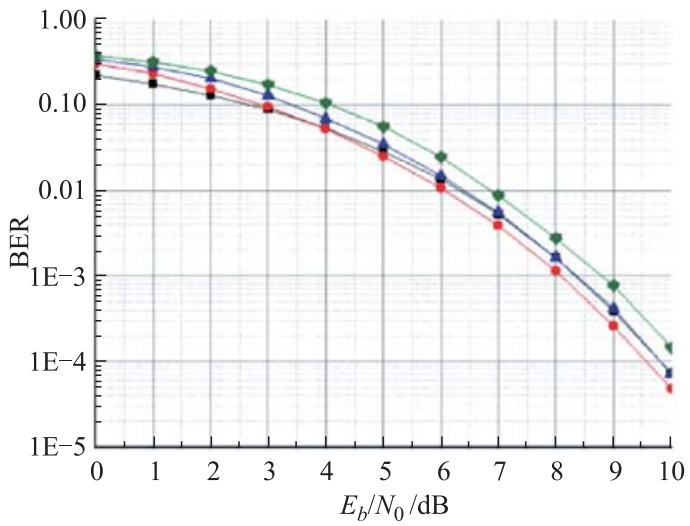

(a) BER curve

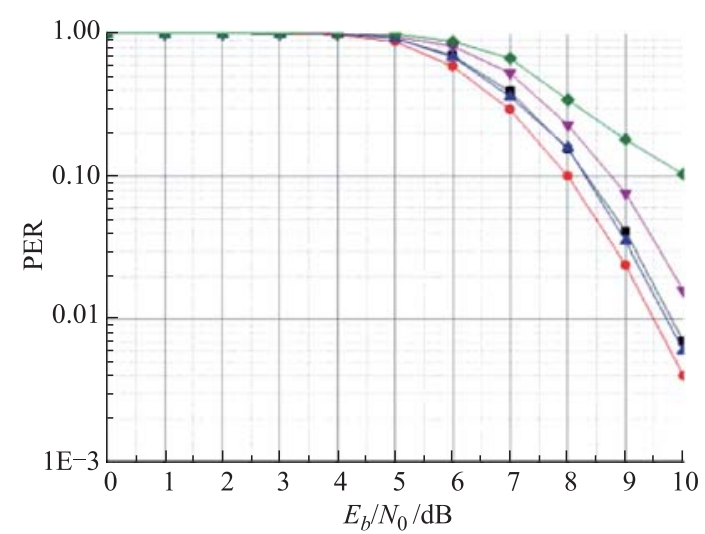

(b) PER curve

$\bullet$ : Reference scheme in [8]; $\leftarrow$ : Proposed scheme with $N=14$;

$\rightarrow$ : Proposed scheme with $N=20 ; \leftrightarrow \bullet$ : Proposed scheme with $N=8$;

$\rightarrow-$ : Proposed scheme with $N=10$.

Fig. 2 Performance of the proposed NSD scheme with different implicit phase memories
The carrier phase of the received signal is random with uniform distribution in the interval $[0,2 \pi)$. Performance of the reference scheme in [8] is also shown for comparison. With the growth of the implicit phase memory $N$, performance of the proposed scheme is improved while the complexity increases. When the implicit phase memory is $N=14$, performance of the proposed scheme approaches that of the reference scheme.

Robustness of the proposed scheme $(N=14)$ and the reference scheme in [8] are illustrated in Fig. 3, where the BER and PER curves versus the normalized frequency offset $f_{d} T_{b}$ for $E_{b} / N_{0}=10 \mathrm{~dB}$ are shown. The case of no interference is under consideration. Performance of the reference scheme degrades fast with the increase of the normalized frequency offset. And the performance degradation of the proposed scheme is caused by the suboptimality of the matched-filter front end in the presence of frequency offsets. When $f_{d} T_{b}=0.05$, the PER of the reference scheme approaches one while the PER of the proposed scheme is about 0.02 . With the growth of the normalized frequency offset, the performance of the proposed scheme degrades slowly. It is obvious that the proposed scheme is more tolerant in larger frequency offset than the reference scheme.

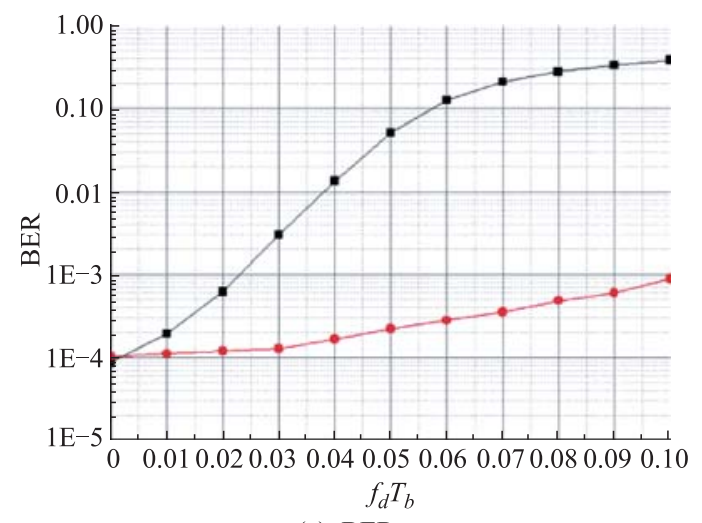

(a) BER curve

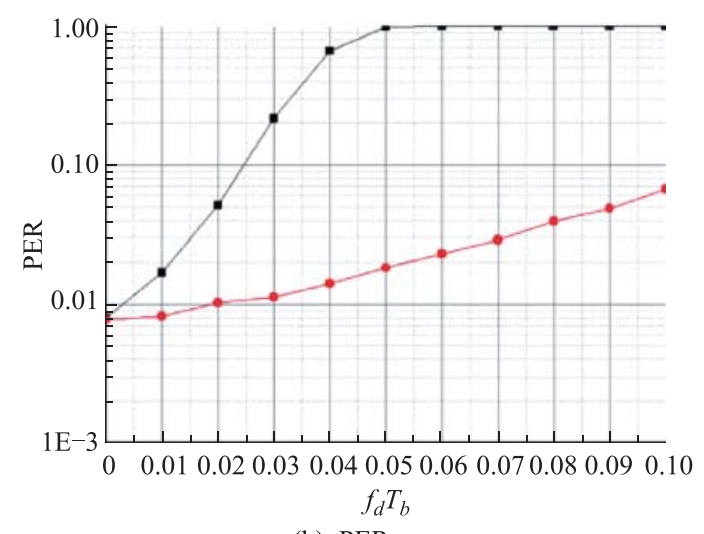

(b) PER curve

: Reference scheme in [8]; $\rightarrow$ : Proposed scheme with $N=14$.

Fig. 3 Robustness of schemes under different $f_{d} T_{b}$ 
In Fig. 4, we consider the case of single interference in addition to the interested signal under the condition that the signal-to-interference power ratio (SIR) is $7 \mathrm{~dB}$. BER and PER curves of the proposed scheme $(N=14)$ and the reference scheme in [8] are shown with different frequency offsets of the interested signal. The frequency offset of interference is a random variable with uniform distribution in the interval $[-4 \mathrm{kHz}, 4 \mathrm{kHz}]$. Fig. 4 illustrates that the proposed scheme excels over the reference scheme in the aspect of robustness to high frequency offset. The reference scheme outperforms the proposed scheme when the interference exists and $f_{d}=0 \% R_{b}$. However, with the increase of $f_{d}$, the performance loss of the reference scheme is becoming serious. The performance of the proposed scheme with $f_{d}=4 \% R_{b}$ approaches that of the reference scheme with $f_{d}=1 \% R_{b}$.

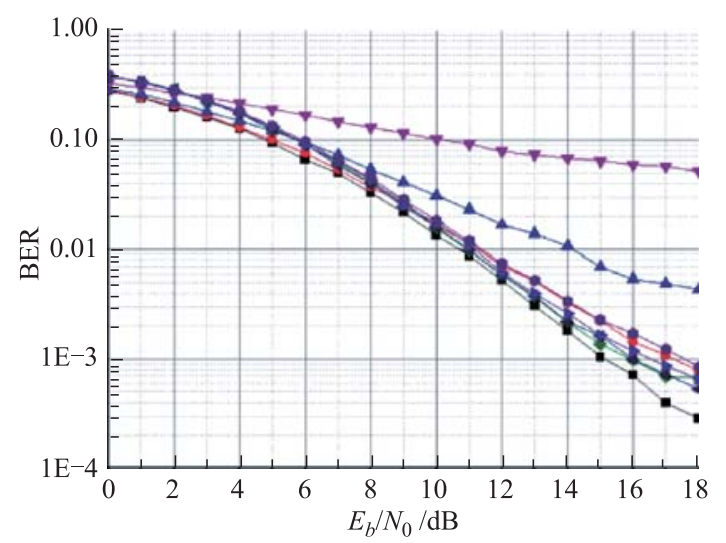

(a) BER curve

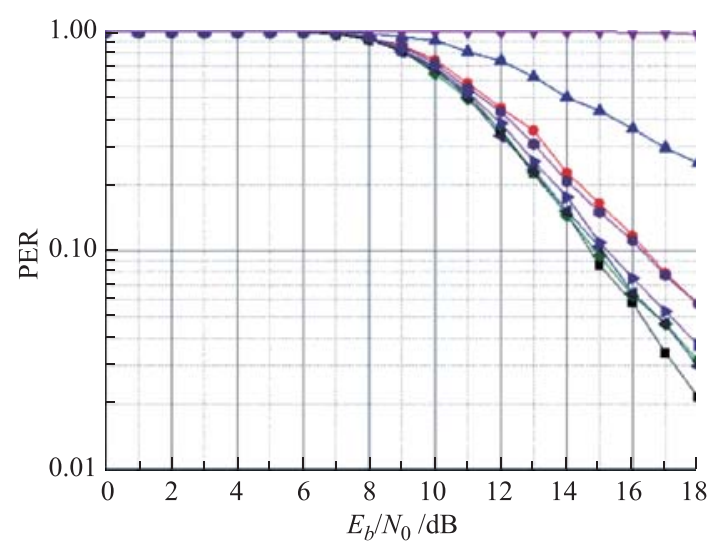

(b) PER curve

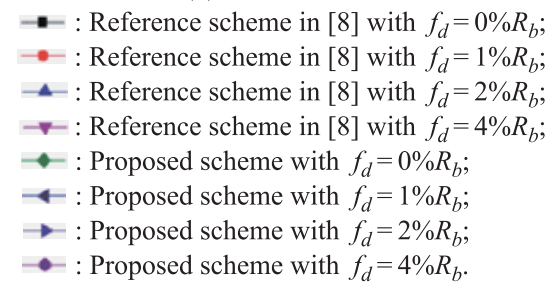

Fig. 4 Performances of the proposed scheme with different $f_{d}$ under $\operatorname{SIR}=7 \mathrm{~dB}$
We consider the case of two interferences in addition to the interested signal in Fig. 5 under the condition that $E_{b} / N_{0}=15 \mathrm{~dB}$. BER and PER curves versus SIR of the proposed scheme $(N=14)$ and the reference scheme in [8] are shown with different frequency offsets of the interested signal, respectively. The frequency offset of interference, a random variable with uniform distribution, is in the interval $[-4 \mathrm{kHz}, 4 \mathrm{kHz}$. Fig. 5 illustrates again that the proposed scheme has high robustness to frequency offset compared with the reference scheme in [8].

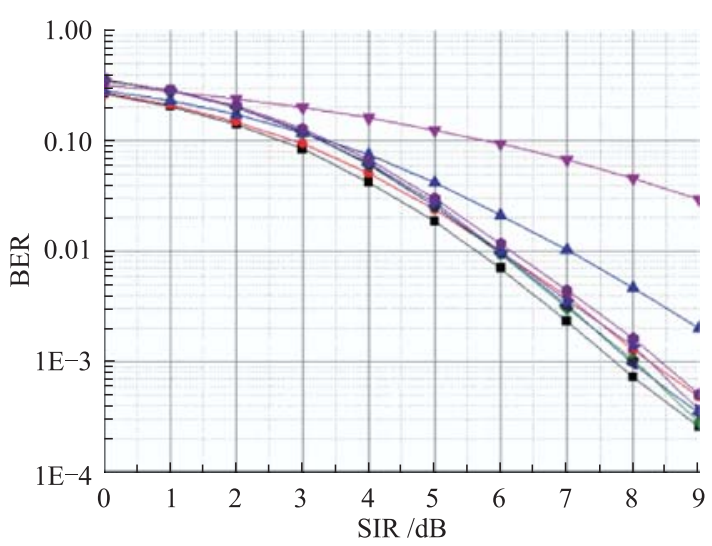

(a) BER curve

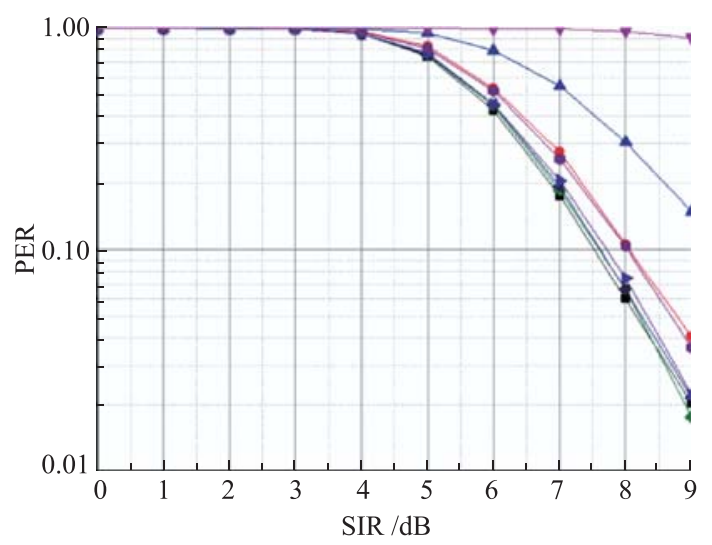

(b) PER curve

$$
\begin{aligned}
& \text { - : Reference scheme in [8] with } f_{d}=0 \% R_{b} \text {; } \\
& \rightarrow: \text { Reference scheme in [8] with } f_{d}=1 \% R_{b} \text {; } \\
& \checkmark \text { : Reference scheme in [8] with } f_{d}=2 \% R_{b} \text {; } \\
& \rightarrow \text { : Reference scheme in [8] with } f_{d}=4 \% R_{b} \text {; } \\
& \rightarrow \text { : Proposed scheme with } f_{d}=0 \% R_{b} \text {; } \\
& \leftarrow \text { : Proposed scheme with } f_{d}=1 \% R_{b} \text {; } \\
& \rightarrow \text { : Proposed scheme with } f_{d}=2 \% R_{b} \text {; } \\
& \rightarrow \text { : Proposed scheme with } f_{d}=4 \% R_{b} \text {. }
\end{aligned}
$$

Fig. 5 Performances of the proposed scheme with different $\boldsymbol{f}_{d}$ un$\operatorname{der} E_{b} / N_{0}=15 \mathrm{~dB}$

\section{Conclusions}

This paper proposes an NSD scheme for satellite-based AIS signals in the presence of frequency offset and white Gaussian noise channel. The proposed scheme, based on 
the Viterbi detection, is characterized by high robustness to frequency offset up to $5 \%$ of the symbol rate. The complexity of the proposed detection scheme has been reduced by technology for state-complexity reduction. Simulation results prove that the proposed scheme has high robustness to frequency offset compared with the reference scheme in [8].

\section{References}

[1] J. E. Hichs, J. S. Clark, J. Stocker, et al. AIS/GMSK receiver on FPGA platform for satellite application. Proc. of the SPIE Digital Wireless Communication VII and Space Communication Technologies, 2005: 403-414.

[2] M. A. Cervera, A. Ginesi. On the performance analysis of a satellite-based AIS system. Proc. of the 10th International Workshop on Signal Processing for Space Communications, 2008: $1-8$.

[3] D. C. Smith, D. J. Nelson. A comparison of two methods for demodulating a target AIS signal through a collision with an interfering AIS signal. Proc. of the SPIE Mathematics for Signal and Information Processing, 2009: 1-12.

[4] M. Cervera, A. Ginesi, K. Eckstein. Satellite-based vessel automatic identification system: a feasibility and performance analysis. International Journal of Satellite Communications and Networking, 2011, 29(2): 117-142.

[5] M. J. Gallardo, U. Sorger. Coherent receiver for AIS satellite detection. Proc. of the 4th International Symposium on Communications, Control and Signal Processing, 2010: 1-4

[6] R. Prevost, M. Coulon, D. Bonacci, et al. Cyclic redundancy check-based detection algorithms for automatic identification system signals received by satellite. International Journal of Satellite Communications and Networking, 2013, 31(4): $157-$ 176.

[7] G. Colavolpe, R. Raheli. Noncoherent sequence detection of continuous phase modulations. IEEE Trans. on Communications, 1999, 47(9): 1303-1307.

[8] P. Burzigotti, A. Ginesi, G. Colavolpe. Advanced receiver design for satellite-based automatic identification system signal detection. International Journal of Satellite Communications and Networking, 2012, 30(2): 52-63.

[9] H. Zhou, T. Ban, J. Wang. A cyclic redundancy check-based non-coherent receiver for automatic identification system signals. International Journal of Satellite Communications and
Networking, 2016, 34(2): 251-261.

[10] Y. L. Huang, K. D. Fan, C. C. Huang. A fully digital noncoherent and coherent GMSK receiver architecture with joint symbol timing error and frequency offset estimation. IEEE Trans. on Vehicular Technology, 2000, 49(3): 863-874.

[11] G. Colavolpe, R. Raheli. Detection of linear modulations in the presence of strong phase and frequency instabilities. IEEE Trans. on Communications, 2002, 50(10): 1617-1626.

[12] G. Colavolpe, R. Raheli. Reduced-complexity detection and phase synchronization of CPM signals. IEEE Trans. on Communications, 1997, 45(9): 1070 - 1079.

[13] International Telecommunication Union. Technical characteristics for an automatic identification system using time division multiple access in the VHF maritime mobile band. Recommendation ITU-R(0). M.1371-4, 2010.

[14] U. Mengali, M. Morelli. Decomposition of $M$-ary CPM signals into PAM waveforms. IEEE Trans. on Information Theory, 1995, 41(5): $1265-1275$.

[15] G. Colavolpe, R. Raheli. Noncoherent sequence detection. IEEE Trans. on Communications, 1999, 47(9): 1376-1385.

\section{Biographies}

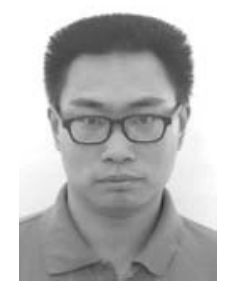

Haosu Zhou was born in 1989. He received his B.S. degree in School of Electronic and Optical Engineering, Nanjing University of Science and Technology, Nanjing, China, in 2012. Since September 2012, he has been a Ph.D. student in School of Electronic and Optical Engineering in the same university. His main research interests include digital communications and automatic iden-

tification system

E-mail: zhouhaosu@sina.com.cn

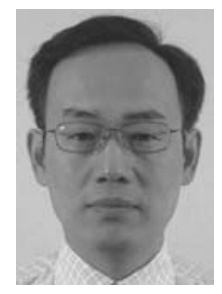

Jianxin Wang was born in 1963. He received his M.S. and Ph.D. degrees from Nanjing University of Science and Technology, in 1987 and 1999, respectively. Since 1987, he has been with School of Electronic and Optical Engineering at Nanjing University of Science and Technology as a teaching assistant, lecturer, and associate professor, and since 2001 as a professor. His main research areas are communications signal processing and software radio.

E-mail: wangjxin@njust.edu.cn 